Ann. Zootech., I97I, 20 (I), I07-IIO

\title{
ÉTUDE D'UN DIAGNOSTIC DE GESTATION PAR ULTRASONS ET EFFET DOPPLER, CHEZ LA BREBIS
}

\author{
M.-J. BOSC \\ Station de Recherche sur la Physiologie de la Reproduction, \\ Centre de Recherches de Tours, I. N.R.A. \\ 37 - Nouzilly
}

Chez la Brebis, il est possible d'obtenir une gestation au cours de la saison d'anœstrus (THIMONIER et al., I968) ; mais, à la suite des différents traitements nécessaires, un certain nombre de brebis ne sont pas gestantes et l'état de ces dernières ne peut pas être connu avant la saison normale de reproduction. Le fait de pouvoir séparer le plus tôt possible les brebis gestantes des non. gestantes s'avère donc nécessaire pour adapter l'alimentation des brebis à leur état et pour remettre les brebis vides à la reproduction sans attendre la saison sexuelle. Pour cela, nous avons entrepris l'étude d'un diagnostic de gestation combinant les propriétés des ultrasons et le principe de l'effet Doppler. A cet effet, nous nous sommes appuyés sur les travaux de Fraser et Robertson (1968) de Hulet (1969) et de Lindahl (1968).

\section{MATÉRIEL ET MÉTHODES}

Deux essais ont été réalisés sur des brebis de race Préalpes. Au cours du premier, 36 brebis inséminées artificiellement en novembre 1969, ont été examinées plusieurs fois à des stades de gestation bien définis. Pour les II brebis du deuxième essai, inséminées après synchronisation et induction de l'ovulation au printemps I970, il n'y eut qu'un examen par animal, examen limité à 5 minutes.

Le principe du diagnostic repose sur l'effet Doppler. Des ultrasons émis à partir d'une source fixe sont réfléchis par tout ensemble mobile tels que le cœur ou les globules rouges du sang; ils subissent alors une modification de fréquence proportionnelle à la vitesse de déplacement de l'ensemble réflecteur. On dispose d'une sonde exploratrice à partir de laquelle sont émis et reçus les ultrasons. Cette sonde est reliée à un appareil qui convertit la différence de fréquence entre les ultrasons incidents et réfléchis en signaux audibles caractéristiques des ensembles réflecteurs.

L'appareil que nous avons utilisé a été décrit pour la débitmétrie par Pourcelor (I966) : les ultrasons sont émis à une fréquence de $3 \mathrm{Mhz}$ avec une puissance inférieure à $200 \mathrm{~mW} / \mathrm{cm}^{2}$. Le critère du diagnostic de gestation retenu est la reconnaissance et l'écoute du rythme cardiaque fœtal, soit au niveau du cœur du fœtus, soit au niveau d'une de ses artères. 


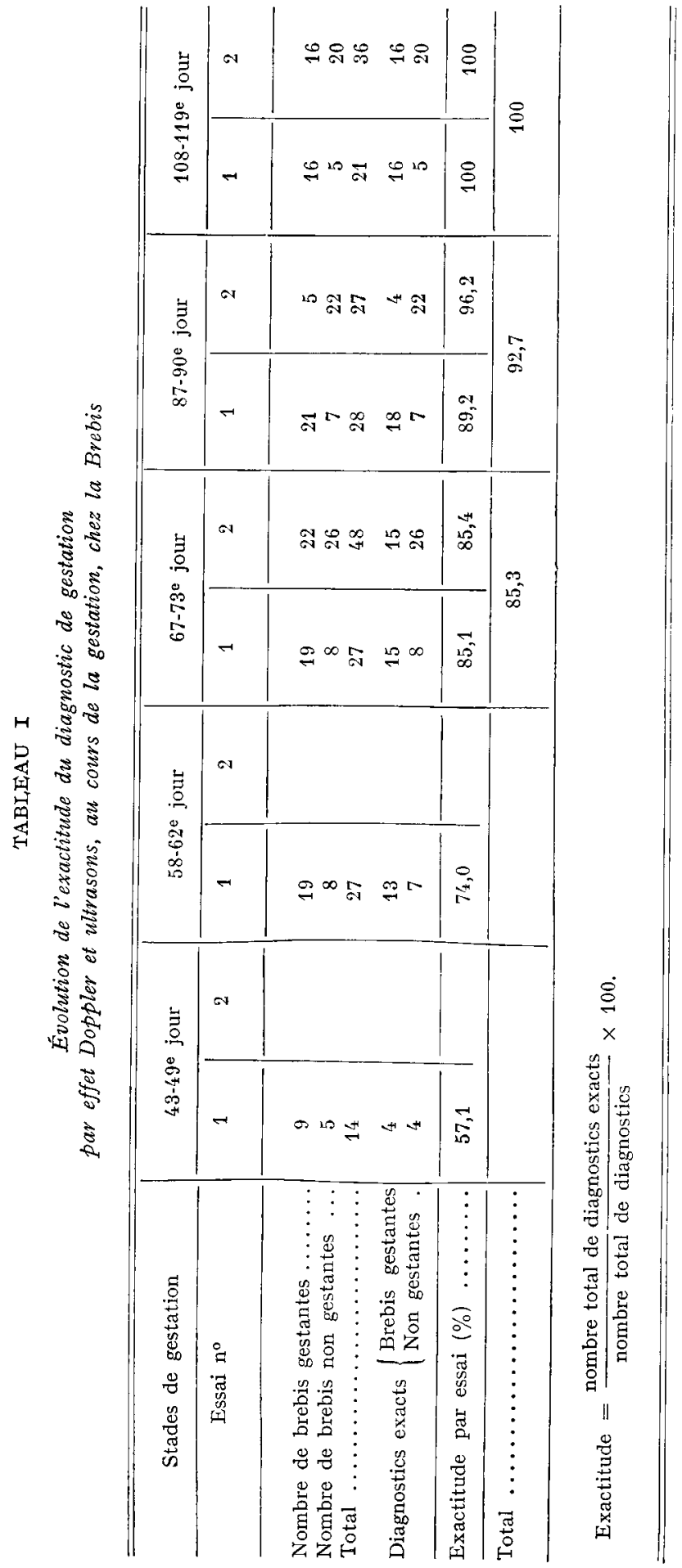


Chaque examen se fait en appliquant la sonde exploratrice sur une zone abdominale médiane légèrement antérieure au pis. La sonde est fortement maintenue sur la peau de manière à pouvoir faire varier dans la cavité abdominale l'angle solide des ultrasons incidents, tout en conservant un bon contact sonde-peau.

Afin d'obtenir ceci, la zone abdominale d'examen est rasée et enduite d'une pâte lubrifiante soluble à l'eau. Pendant l'examen, la brebis est maintenue debout par un aide.

\section{RÉSULTATS}

Les diagnostics ainsi portés ont été comparés aux résultats de l'agnelage ; les données obtenues de cette façon sont regroupées dans le tableau I où nous avons présenté l'exactitude des diagnostics par le rapport de ceux qui se sont révélés exacts sur le nombre total de ceux qui ont été effectués. Pour les deux essais, et pour les mêmes stades de gestation, l'exactitude est identique ; elle est faible à un mois et demi puisqu'elle n'atteint que 57, I p. roo; à 7o jours de gestation, elle augmente à $85,3 \mathrm{p}$. roo ; à trois mois elle est égale à 92,7 p. Ioo pour atteindre I00 p. I0O I ro jours après l'insémination.

Dans le tableau 2, nous avons regroupé les diagnostics portés sur les brebis gestantes en tenant compte du nombre d'agneaux produits à la naissance. 75,0 p. 1oo des brebis portant un seul fotus sont diagnostiquées gestantes au $7^{\circ}$ jour de gestation, $84,6 \mathrm{p}$. I oo à trois mois alors que 7 I,4 p. Ioo et 78,5 p. Ioo des brebis portant plus d'un fœtus le sont aux mêmes moments. Ces différences ne sont pas significatives.

TABLEAU 2

Influence du nombre d'agneaux sur l'exactitude du diagnostic de gestation par effet Doppler et ultrasons

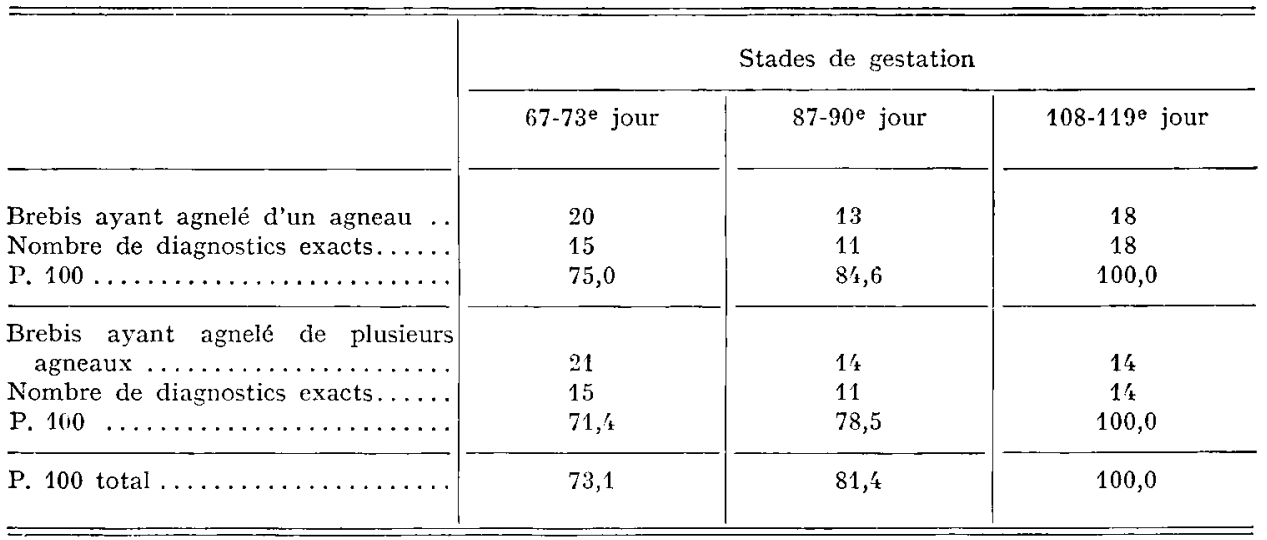

\section{DISCUSSION}

La détection de la vie foetale est possible après un mois et demi de gestation, avec cette technique, puisque 4 brebis gestantes ont été diagnostiquées à ce moment. Ceci rejoint les observations de LINDAHL (I969). HELPER (I970) signale la possibilité de détecter le cœur fœtal dès le $3^{2}$ jour de la gestation, chez la Chienne, alors que chez la Femme, il faut attendre $7^{\circ}$ jours environ (BIsHop, I966).

L'exactitude du diagnostic augmente au fur et à mesure qu'on approche de la parturition 
et on conçoit aisément qu'elle soit fonction du développement du fœetus et du placenta. Lorsqu'on ne considère que les brebis gestantes, les taux de diagnostics exacts sont inférieurs à ceux qui sont obtenus sur toutes les brebis aux mêmes moments, ce qui est conforme au critère retenu : l'écoute d'un rythme cardiaque fotal. Le fait de limiter le temps d'examen de chaque brebis ne modifie pas la précision du diagnostic puisque les résultats obtenus dans les deux essais sont sensiblement les mêmes (tabl. I). De plus, l'évolution et la valeur du diagnostic sont identiques pour les brebis portant un fotus et pour celles portant des jumeaux, ce qui diffère des observations de LINDAHL (I968) et de HULET (I969) qui obtiennent une précision plus élevée sur ce dernier type de brebis. Au cours de nos deux essais, nous n'avons pas tenté de détecter le nombre de foetus par brebis. Nous pensons en effet que cela est rendu difficile par la variabilité même du rythme cardiaque fotal (Melchion et al., I970 ; Too et al., i 966) ce que confirment les résultats de WiLson et NewToN (1969).

Cette technique est d'un emploi facile et rapide, mais pour obtenir une précision suffisante des diagnostics ainsi portés, il faut réaliser les examens après 7o jours de gestation.

Recu pour publication en décembre 1970.

\section{SUMMARY}

\section{STUDY OF A PREGNANCY DIAGNOSIS IN THE EWE BY MEANS OF AN ULTRASONIC DOPPLER INSTRUMENT}

A pregnancy diagnosis based upon a technique combining ultrasonic properties with the Doppler effect was carried out on 147 ewes of the Pvéalpe breed. The diagnoses performed were compared with the lambing data. The results showed that the accuracy of all the diagnoses concerning pregnant and nonpregnant ewes varied according to the stage cf pregnancy at which the diagnoses were established. The accuracy was $57, \mathbf{I}$ p. Ioo one month and a half after the insemination, $85,3 \mathrm{p}$. I 00 on the 7 oth day of pregnancy and Ioo $\mathrm{p}$. Ioo on the I Ioth day. The accuracy of the diagnoses performed solely on pregnant ewes was a little lower than that obtained on all the ewes, and, in our study, it did not depend upon the number of lambs carried by the ewe.

\section{RÉFÉRENCES BIBLIOGRAPHIQUES}

Bishop E. H., i966. Obstetrics use of the ultrasonic motion sensor. Am. J. Obst. Gynecol., 96, 863-867.

Fraser A. P., Robertson J. G., I968. Pregnancy diagnosis and detection of foetal life in sheep and pigs by an ultrasonic method. Brit. Vet. J., 124, 239-244.

Helper L. C., 1970. Diagnosis of pregnancy in the bitch with an ultrasonic Doppler instrument. $J$. Am. Vet. Med. Assoc., 156, 60-62.

Hulet C. V., I969. Pregnancy diagnosis in the ewe using an ultrasonic Doppler instrument. J. anim. Sci, 28, 44-47.

Lindahl I. L., x968. Pregnancy diagnosis in ewes in continual breeding. J. anim. Sci., 27, r5r I (Abstr.).

LindhAL I. L., I969. Comparison of ultrasonic techniques for the detection of pregnancy in ewes. $J$. Reprod. Fert., 18, II7-I20.

Melchior J., Distel R., Bernard N., I969. Le rythme cardiaque du fœetus humain. J. Physiol., 61, Suppl. II, 35I.

Pourcelot L., George P., Fourcade C., Guillaud C., 1966. Débitmètre sanguin à effet Doppler. Électronique Médicale, 35, I3-I7.

Thimonier J., Mauléon P., Cognie Y., Ortavant R., ig68. Déclenchement de l'cestrus et obtention de la gestation pendant l'anostrus post-partum, chez les brebis, à l'aide d'éponges vaginales imprégnées d'acétate de fluorogestone. Ann. Zootech., 17, 257-273.

Too K., Kanagawa H., Kawata K., I965. Fetal electrocardiogram in dairy cattle. I. Fundamental studies. Jap. J. Vet. Res., 13, 7I-9I.

Wilson I. A. N., Newton J. E., Ig69. Pregnancy diagnosis in the ewe a method for use on the farm. Vet. Rec., 84, 356-358. 